\title{
British Association for the Study of the Liver
}

The 1996 Annual Meeting of the British Association for the Study of the Liver was held at the Postgraduate Medical Centre, Queen Elizabeth Hospital, Birmingham on 8-9 February 1996. The 18 abstracts selected for presentation by the Programme Committee are printed below.

Mutations in a putative hepatitis B HLA A1 CTL epitope affect HLA binding and correlate with disease severity

C J HEALEY, J CHRISTIE, M E LAI, V CERUNDOLO, R W CHAPMAN, W M C ROSENBERG * (Nuffield Department of Clinical Medicine, Oxford and Ospedale Microcitemico, Cagliari, Italy) A vigorous peripheral blood cytotoxic T-cell (CTL) response is associated with viral clearance in acute HBV infection but intrahepatic CTL may mediate hepatocyte damage in chronically infected subjects. Escape from immune detection is thought to lead to persistent infection. In a family chronically infected with HBV with differing outcomes we have studied the effect of mutation within a core HLA-A1 restricted CTL epitope on HLA-A1 binding and disease course.

HBV core protein was sequenced in viral isolates from HLA-typed family members. Synthetic peptides based on the HBV sequences were tested for binding to HLA-A1 molecules in vitro using an HLA class I assembly assay. Binding was correlated with disease course.

One patient with a mutation in the A1 epitope, which abrogates HLA-A1 binding and three who lack HLA-A1 had no liver disease. Two patients with mutations that increased binding and one patient without mutations developed chronic liver disease.

A benign course of $\mathrm{HBV}$ infection may result from failure of CTL recognition due to mutations in HLA binding residues that abrogate binding.

\section{CTL epitope mapping in HCV using HLA assembly and CTL assays}

C J HEALEY, $S$ MCADAM, M PLEBANSKI, $R$ W CHAPMAN, W M C ROSENBERG ${ }^{\star}$ (Nuffield Department of Clinical Medicine, Oxford) Cytotoxic $\mathrm{T}$ lymphocytes (CTL) clear acute viral infections but may facilitate viral persistence through selection of variants that escape immune detection. Immune escape may arise by loss of peptide binding to HLA molecules or through failure of CTL recognition. We have established assays of HLA binding and CTL recognition to map HCV immune epitopes.

(a) The HCV genome was scanned for HLA-B7 binding motifs ( $x$ PxxxxxxL). Nine HLA-B7 peptides and seven control HLA-A2 peptides were each incubated with radiolabelled HLA molecules. Binding affinity was determined by isoeletric immune precipitation with the conformational specific Mab (W/623). (b) CTL were cultured from peripheral blood (with peptide, IL-2, IL-7, and $K L H$ ) and tested for their ability to lyse autologous peptide-pulsed target cells.

All control HLA-A2 peptides bound. Seven of nine candidate $B 7$ peptides bound to HLA-B7, of which one HLA-B7 peptide, identifying a new epitope, and an HLA-A2 control epitope were recognised by patient derived CTL.
We have used an HLA assembly to identify seven new HLA-B7 HCV epitopes and have shown CTL recognition of at least one.

An analysis of factors affecting posttransplant lymphoproliferative disorder (PTLD) following orthotopic liver transplantation (OLT)

M MCCARTHY ${ }^{1}$, J RAMAGE ${ }^{1}$, E GANE $^{1}$, B PORTMANN $^{1}$, M RELA $^{2}$, N HEATON $^{2}$, A PAGLIUCA ${ }^{3}$, G MUFTI $^{3}$, ROGER WILLIAMS ${ }^{1}$ (Institute of Liver Studies $^{1}$, Liver Transplant Surgical Service ${ }^{2}$ and Department of Haematology ${ }^{3}$, King's College Hospital, London SE5 9RS) Over a 15 year period at this unit, 10 adult liver allograft recipients (six females, four males) developed PTLD three to 72 months post-OLT (median 22.2 months), an overall prevalence of $2 \cdot 1 \%$. Mean age was $47 \cdot 1$ years (range 24 to 56). Sites of primary lesions were: liver graft three of 10 , kidneys two of 10 , ovaries one of 10 , brain one of 10 , lungs two of 10 , pancreas one of 10 , bone marrow two of 10 , and lymph nodes four of 10 . All tumours were B-cell lymphomas: six lymphoblastic, three immunoblastic, and one Hodgkin's lymphoma. Clonality was assessed by both immunohistochemistry and gene rearrangement studies: four were monoclonal, three polyclonal, and three undetermined. EpsteinBarr virus (EBV) serology (EBNA, VCR antigens), EBV PCR, and in situ hybridisation (ISH) were performed: six of 10 were positive for EBNA antigens and seven of 10 had EBV detected in lymphoma tissue by PCR or ISH. Monoclonal tumours were treated with systemic chemotherapy, polyclonals with combinations of immunosuppression withdrawal and acyclovir. Mortality is $60 \%$ and those alive have a mean survival of 720 days (range 600 to 1440 ).

PTLD is presenting earlier post-OLT and continues to have a high mortality. Exposure to EBV plays a significant part in tumour development and efforts must be directed towards reducing the risk of exposure to the virus early post-OLT.

The haemochromatosis gene lies more than $3 \mathrm{Mb}$ telomeric to HLA-A

J D SHEARMAN, J J POINTON, C STONE, A MERRYWEATHER-CLARKE, W M C ROSENBERG $^{\star}, \mathrm{K} \mathrm{J} \mathrm{H}$ ROBSON for the UK Haemochromatosis Consortium (Nuffield Department of Clinical Medicine and MRC Haematology Unit, Oxford) The molecular defect in haemochromatosis (HC) remains undefined. Genetic linkage analysis has placed the gene close to HLA-A on chromosome six and is a candidate for positional cloning. The candidate region for the gene covers several megabases, extending from HLA-F to D6S299. The disease is strongly associated with the microsatellite marker D6S105, which maps telomeric to HLA-A within his region. We are analysing linkage of $\mathrm{HC}$ to newly generated microsatellite markers close to D6S105 to narrow the candidate region in an attempt to identify the gene.

We have constructed a yeast artificial chromosome (YAC) contig extending $1.5 \mathrm{Mb}$ telomeric to HLA-A beyond the telomeric limit of the MHC and towards D6S105. A second contig centred on D6S105 spanning $3 \mathrm{Mb}$ does not overlap the proximal contig. New microsatellites isolated from this contig all show strong allelic association with the disease and locate a peak of association $700 \mathrm{~kb}$ telomeric to D6S105. Although haemochromatosis shows strong linkage to HLA-A these studies show that the gene lies more than $3 \mathrm{Mb}$ telomeric to this locus. These findings will permit identification of the disease locus.

Hepatic expression of gelatinase $A$ is increased during progressive liver fibrosis

R C BENYON, S PAWLEY, C J HOVELL, J P IREDALE, M J P ARTHUR (University Medicine, Southampton General Hospital, Southampton) Gelatinase A released from hepatic stellate cells (HSC) activated after liver injury may degrade normal basement membrane and promote fibrogenesis by disturbing HSC and hepatocyte function. We have examined this hypothesis by quantifying hepatic expression of gelatinase $\mathrm{A}$ in two rat models of progressive fibrosis. Hepatic fibrosis was induced by bile duct ligation (BDL) or $\mathrm{CCl}_{4}$ administration twice weekly. Some rats were given $\mathrm{CCl}_{4}$ for four weeks then left to recover for four weeks. RNAase protection assay quantified gelatinase mRNA content of total liver RNA. Gelatinase mRNA was increased at 24 hours (383\% of control) and three days $(400 \%)$ after BDL. In the $\mathrm{CCl}_{4}$ model, gelatinase mRNA was $157 \%$ of control at 24 hours, $595 \%$ (72 hours), $295 \%$ (one week), and $289 \%$ (four weeks). However, this expression fell during $\mathrm{CCl}_{4}$ recovery. At 0 day recovery, gelatinase mRNA was $537 \%$ of untreated controls, falling to $201 \%, 210 \%, 111 \%$, and $148 \%$ at three, seven, 14 , and 28 days recovery. HSC isolated from normal rat liver showed increasing expression of gelatinase mRNA during activation in vitro by culture on plastic. By northern blotting, gelatinase/ $\beta$-actin mRNA ratio was 0.099 in freshly isolated HSC and 0.322, 0.758, 0.903 at five, seven, and 10 days of culture respectively. We conclude that progressive liver fibrosis is associated with increased hepatic gelatinase mRNA expression and that activated HSC may be an important source of this enzyme.

Analysis of the TIMP-1 promoter activity in freshly isolated and activated hepatic stellate cells

M J BAHR, J P IREDALE, I M CLARK ${ }^{\star}$, M J P ARTHUR, D A MANN* (University Medicine, ^University Clinical Biochemistry, Southampton General Hospital, Southampton, ${ }^{\star}$ Rheumatology Research Unit, Addenbrooke's Hospital, 
Cambridge) Activation of hepatic stellate cells (HSC) is central to the process of hepatic fibrosis. When activated, HSC produce significant amounts of tissue inhibitor of metalloproteinases-1 (TIMP-1), which downregulates the matrix-degrading activity of many matrix metalloproteinases. The aim of this study was to examine the regulation of the promoter of the human TIMP-1 gene in HSC. We therefore adapted several methods of transient DNA transfection into eukaryotic cells for the use in HSC. Electroporation was a very reproducible method of transfection in activated HSC. However, because of the high numbers of cell death no transfection of freshly isolated HSC could be obtained. Calcium phosphate mediated transfection worked well for both freshly isolated and activated HSC. Different promoters including viral (SV40, HSV thymidine kinase), eukaryotic ( $\beta$-actin), and artificial sequences (AP-1, NFKB) linked to CAT reporter genes were found to be active in HSC. The $5^{\prime}$ region of the human TIMP1 gene was isolated, sequenced, and cloned into the pBLCAT3 vector. TIMP-1 promoter activity was found upregulated in activated HSC. By the use of promoter truncations regulatory sequences have been identified. In conclusion, TIMP-1 activity in HSC is regulated at the promoter level.

\section{Identification of a potential novel regula-} tory protein in Kupffer cell activation

D HOU, S L FRIEDMAN ${ }^{\star}$, A LALAZAR, M J P ARTHUR, P J WINWOOD University Medicine, Southampton General Hospital, Southampton, *Liver Center Laboratory, University of California, San Francisco) In liver injury Kupffer cells (KC) undergo phenotypic changes in response to a programme of gene induction termed activation. Although central to the pathogenesis of liver injury, little is known about transcriptional regulation of $\mathrm{KC}$ activation.

cDNAs that are induced in rat KC (freshly isolated) two hours after administration of $\mathrm{CCl}_{4}$, were identified by a subtractive hybridisation/differential screening approach. cDNAs deemed activation specific were cloned into suitable vectors for DNA sequencing and expression studies. Full length cDNAs were isolated using marathon RACE.

One cDNA identified, $\mathrm{KC} 2$, has s 270 bp motif (78\% homology) common to human ETO (MTG8) protein, a putative zinc finger transcription factor in myeloid cell types. Marathon RACE yielded a $3 \mathrm{~kb} \mathrm{cDNA}$ in the $3^{\prime}$ direction with a poly A tail. Sequence data obtained to date show no homologies to known genes (Blast). RNAse protection analysis showed induction of the transcript of $\mathrm{KC} 2$ in freshly isolated $\mathrm{KC}$ after $\mathrm{CCl}_{4}$, peaking at 12 hours. Northern analysis of whole liver RNA, using the $3 \mathrm{~kb}$ RACE product as a probe, detected a specific transcript maximal at 24 hours, not identified in normal liver RNA.

We have identified a novel cDNA in $\mathrm{KC}$, which is induced early after $\mathrm{CCl}_{4}$ liver injury. Sequence analysis shows homology to a myeloid transcription factor, ETO.

Interaction between cytokine activated killer cells and human intrahepatic biliary epithelial cells: modulation by ICAM-1 blockade

M P LEON, J A KIRBY, P GIBBS, M THICK, M F BASSENDINE (Departments of Medicine and
Surgery, University of Newcastle upon Tyne) Human intrahepatic biliary epithelial cells (HIBEC) constitute a major immunological target during liver allograft rejection and primary biliary cirrhosis. This has been attributed to their capacity to express immune cell ligands during inflammation. Initial experiments showed that cultured HIBEC after stimulation with pro-inflammatory cytokines increased their expression of ICAM-1 and their capacity to bind mononuclear cells.

Standard ${ }^{51} \mathrm{Cr}$-release assays were used to assess the capacity of cytotoxic effector cells to damage cultured HIBEC; IL-2 activated natural killer (LAK) cells were used in this experiment to bypass the requirement for antigen specificity. Cultured HIBEC were stimulated for four days with pro-inflammatory cytokines and were loaded with ${ }^{51} \mathrm{Cr}$ for one hour; LAK cells were then added for four hours. Anti-ICAM-1 antibody was added to come cultures to modulate LAK cell adhesion to ICAM-1 expressing HIBEC.

Addition of LAK cells at an increasing effector:target (E:T) ratios caused an increasing release of ${ }^{51} \mathrm{Cr}$; a ratio of 200:1 produced a maximum specific ${ }^{51} \mathrm{Cr}$ release of $38 \%$ $(p<0.001)$. Anti-ICAM-1 produced a significant reduction in the adhesion of LAK cells to stimulated HIBEC and also in the amount of ${ }^{51} \mathrm{Cr}$ released from HIBEC.

These results show that the capacity of HIBEC to upregulate their expression of ICAM-1 during inflammation is associated to their susceptibility to lytic damage by cytotoxic effector cells. These findings suggest a potential option for therapeutic intervention.

Increased expression of monocyte chemotactic protein-1 in alcoholic liver disease

N C FISHER, S C AFFORD, P BRUN, C MORLAND, A KEOGH, J FEAR, S G HUBSCHER, D H ADAMS (Liver Unit, Queen Elizabeth Hospital, Edgbaston, Birmingham B15 2TH) Although alcohol induced inflammation is a major factor in the tissue damage of alcoholic liver disease (ALD) the mechanisms of leucocyte recruitment are unknown. We investigated the expression and regulation of monocyte chemotactic protein-1 (MCP-1), a poten monocyte and T-lymphocyte chemotactic factor, in ALD to discover if MCP-1 is involved in alcohol induced inflammation.

MCP-1 protein and mRNA expression were analysed in liver biopsy specimens using immunohistochemistry and in situ hybridisation. MCP-1 production by human hepatocytes was tested in vitro after exposing hepatocytes to TNF $\alpha$, ethanol or acetaldehyde.

Tissue expression of MCP-1: In normal liver MCP-1 protein and mRNA were detected weakly on endothelium within portal tracts. In alcoholic hepatitis there was intense expression of MCP-1 protein and MRNA in ballooned hepatocytes at areas of inflammation. In alcoholic cirrhosis MCP-1 protein and MRNA were upregulated in leucocytes and endothelium within fibrous septa. Hepatocyte secretion of MCP-1: Untreated hepatocytes or hepatocytes exposed only to ethanol or acetaldehyde did not secrete MCP 1. TNF $\alpha$ treated hepatocytes secreted MCP1 (mean $115 \mathrm{pg} / \mathrm{ml}$ ); concomitant treatment with acetaldehyde augmented the effects of TNF $\alpha$ induced MCP-1 secretion (mean 215 $\mathrm{pg} / \mathrm{ml}$ ); whereas concomitant treatment with ethanol reduced it (mean $44 \mathrm{pg} / \mathrm{ml}$ ).
(1) Expression in areas of inflammatory damage and fibrosis suggests a role for MCP1 in the pathogenesis of ALD. (2) A synergistic effect of acetaldehyde and $T N F \alpha$ on hepatocytes suggests a mechanism for MCP1 upregulation in ALD.

Alcoholic hepatitis is associated with upregulation of redox sensitive neutrophil chemoattractant cytokines

J MALTBY, G BIRD, S WRIGHT, N SHERON (University of Southampton, School of Medicine, Southampton General Hospital) Interleukin 8 (IL-8), a neutrophil chemokine, is increased in alcoholic hepatitis. In this study values of the other major neutrophil chemoattractant protein GRO $\alpha$, together with two monocyte specific chemokines RANTES and MIP1 $\alpha$ were assayed by ELISA in liver homogenates.

\begin{tabular}{lcccc} 
& Number & $\begin{array}{l}G R O \alpha \\
(p g / m l)\end{array}$ & \multicolumn{1}{c}{$\begin{array}{l}\text { RANTES } \\
(p g / m l)\end{array}$} & $\begin{array}{c}\text { MIPI } \alpha \\
(p g / m g\end{array}$ \\
\hline Normal controls & 6 & $11 \cdot 0 \pm 5 \cdot 1$ & $21 \cdot 3 \pm 7 \cdot 0$ & $6 \cdot 0 \pm 2 \cdot 1$ \\
Alcoholic hepatitis & 16 & $45 \cdot 7 \pm 9 \cdot 5$ & $69 \cdot 4 \pm 27 \cdot 7$ & $6 \cdot 0 \pm 1 \cdot 1$ \\
Chronic viral hepatitis & 14 & $16 \cdot 9 \pm 4 \cdot 2$ & $100 \cdot 7 \pm 23 \cdot 1$ & $10 \cdot 5 \pm 2 \cdot 4$ \\
$\begin{array}{l}\text { Other alcoholic liver } \\
\text { disease }\end{array}$ & 9 & $12 \cdot 8 \pm 2 \cdot 2$ & $120 \cdot 2 \pm 44 \cdot 6$ & $7 \cdot 4 \pm 2 \cdot 3$ \\
Other liver disease & 21 & $14 \cdot 4 \pm 2 \cdot 5$ & $103 \cdot 7 \pm 21 \cdot 4$ & $7 \cdot 2 \pm 1 \cdot 2$ \\
\hline
\end{tabular}

GRO $\alpha$ was significantly increased in alcoholic hepatitis compared with normal and disease controls, and values correlated significantly with those of IL-8 $(n=47$, $r=0.57, \mathrm{p}<0.001)$. In contrast, mean values of the monocyte chemokine RANTES were slightly lower in the AH group compared with other disease controls, and values of MIP1 $\alpha$ were not significantly increased in any of the disease groups. These data provide further evidence of a role for redox sensitive neutrophil chemokine activation in the pathogenesis of the unique inflammatory injury associated with alcohol intake.

IgA reactivity to intestinal bacterial isolates in alcoholic liver disease

A C DOWDS, T A POULTON, J D MAXWELI (Division of Medicine and Immunology, St George's Hospital Medical School, London SW17 ORE) Alcoholic liver disease is accompanied by an increase in serum IgA due to increased production by $B$ cells. The antigenic stimulus that determines this response is unknown. We speculate that increased gut permeability in alcoholic liver disease allows intestinal bacterial antigens access to the portal circulation and stimulates IgA production. We investigated the IgA reactivity to a range of gut and non-gut bacterial isolates in alcoholic and other liver diseases using immunoblotting

Eleven of $15(73 \%, \mathrm{p}<0.001)$ alcoholic cirrhotic patients had positive reactivity to Bacteroides, 11 of $13(85 \%, \mathrm{p}<0.001)$ to $E$ coli, and 10 of $13(77 \%, \mathrm{p}<0.05)$ to Clostridium perfringens. No responses were seen in PBC, other liver cirrhotic patients, or alcoholic patients. One of $12(8 \%, p=N S)$ alcoholic cirrhotic patients had positive reactivity to Staphylococcus aureus compared with none of the other groups.

The results show that a high proportion of patients with alcoholic cirrhosis have IgA antibodies to Bacteroides, $E$ coli, and Clostridium perfringens but not to Staphylococcus aureus. These antibodies seem to be specific to alcoholic liver disease. Intestinal bacterial antigens may account for the IgA response in alcoholic liver disease. 
Neutrophil superoxide $\left(\mathrm{O}^{-}{ }_{2}\right)$ and hydrogen peroxide $\left(\mathrm{H}_{2} \mathrm{O}_{2}\right)$ production in patients with acute liver failure (ALF)

M CLAPPERTON, N ROLANDO, L SANDOVAL, ROGER WILLIAMS (Institute of Liver Studies, King's College School of Medicine and Dentistry, Bessemer Road, London SE5 9P7) Neutrophil superoxide and hydrogen peroxide production, essential for bacterial killing, was assessed in ALF patients. Isolated patient neutrophils were stimulated by zymosan opsonised with either pooled control serum (control zymosan) or autologous patient serum (patient zymosan). Control neutrophils were stimulated with control zymosan.

Superoxide: 21 ALF patients (14 paracetamol overdose (POD); seven other aetiologies) and 17 controls were tested. Superoxide was significantly lower for ALF POD neutrophils stimulated either by control zymosan (median $432.00 \mathrm{nmol} / 10^{6}$ cells (range 257-942), $\mathrm{p}<0.05$ ) or patient zymosan (median 542.00 nmol $/ 10^{6}$ cells (range $67-699$ ), $\mathrm{p}<0.05$ ) compared with control neutrophils (median $687 \cdot 00 \mathrm{nmol} / 10^{6}$ cells (range $245-976$ ). The difference in superoxide production between non-POD ALF versus control neutrophils was not significant. Hydrogen peroxide: 12 ALF POD patients and 14 controls were tested. Hydrogen peroxide from ALF neutrophils stimulated with either control zymosan (median $5.80 \mathrm{nmol} / 10^{6}$ cells (range 0-9.62), $\mathrm{p}<0.05$ ) or patient zymosan (median 0.98 nmol $/ 10^{6}$ cells (range $0-12 \cdot 65$ ), $\mathrm{p}<0.005$ ) was significantly less than control neutrophils (median $8.00 \mathrm{nmol} / 10^{6}$ cells (range $2 \cdot 40$ 19.00). The degree of encephalopathy, renal failure, and infection did not influence superoxide or hydrogen peroxide production. Overall, a cellular and opsonic defect leads to reduced superoxide and hydrogen peroxide production in ALF due to POD and could increase susceptibility to infection in ALF patients.

Plasma concentrations and hepatic mRNA expression of transforming growth factor- $\beta 1$ in patients with fulminant hepatic failure

Y MIWA, P M HARRISON, P G LANGLEY, R D HUGHES, ROGER WILLIAMS (Institute of Liver Studies, King's College School of Medicine and Dentistry, Bessemer Road, London SE5 9PF) The impaired liver regeneration seen in some aetiological subgroups of patients with fulminant hepatic failure (FHF) could be due to production of an inhibitory factor like transforming growth factor- $\beta 1$ (TGF- $\beta 1$ ). The aim of this study was to investigate plasma concentrations and hepatic mRNA expression of TGF- $\beta 1$ in patients with FHF. Plasma concentrations of TGF- $\beta 1$ measured by ELISA in FHF patients on admission (median $38.8 \mathrm{ng} / \mathrm{ml}$ ), range 8.4-108 $\mathrm{ng} / \mathrm{ml}, \mathrm{n}=57$ ) were significantly higher than those in control subjects $(23.0$ $\mathrm{ng} / \mathrm{ml}, 8.5-34.9 \mathrm{ng} / \mathrm{ml}, \mathrm{n}=20, \mathrm{p}<0.001)$. Significantly higher plasma concentrations were seen in NANB hepatitis patients $(57.9$ $\mathrm{ng} / \mathrm{ml}, 38 \cdot 8-108 \mathrm{ng} / \mathrm{ml}, \mathrm{n}=10, \mathrm{p}<0.001$ ) compared with paracetamol overdose (POD) patients $(37 \cdot 1 \mathrm{ng} / \mathrm{ml}, 8 \cdot 4-72.5 \mathrm{ng} / \mathrm{ml}, \mathrm{n}=47$, $\mathrm{p}<0.001$ ). In liver tissue, northern blot analysis showed increased mRNA expression of TGF- $\beta 1$ in POD $(n=8, p<0.05)$, but not in NANB hepatitis $(n=6)$, compared with controls $(n=4)$, suggesting that the liver is not the only source of the increased plasma TGF- $\beta 1$ in NANB hepatitis. The mRNA expression of H3 histone, a marker for liver proliferation, was significantly increased in POD $(p<0.05)$ compared with the controls, but not in NANB hepatitis. The increased circulating plasma TGF- $\beta 1$ could be a factor in the impaired liver regeneration and related to poor prognosis in FHF due to NANB hepatitis.

Auxiliary partial orthotopic liver transplantation (APOLT) for acute liver failure (ALF)

S P PEREIRA, M MCCARTHY, A J ELLIS, J WENDON, M RELA ${ }^{\star}$, N HEATON*, ROGER WILLIAMS (Institute of Liver Studies, and ${ }^{\star}$ Liver Transplant Surgical Service, King's College Hospital, Denmark Hill, London SE5 9RS) APOLT holds potential advantages over conventional OLT, but experience of the technique in ALF is limited.

We describe our initial experience in seven patients (four males, three females, mean age 28, range 14-35 years) with ALF (paracetamol three, NANB two, autoimmune one, ecstasy one, who fulfilled $\mathrm{KCH}$ criteria. At listing, the median values for the INR, creatinine and bilirubin were 7 (range 3.4-15), 123 $\mu \mathrm{M}$ (51-389 $\mu \mathrm{M})$, and $320 \mu \mathrm{M}$ (61-648 $\mu \mathrm{M})$, respectively. The reasons for performing APOLT, rather than OLT, were macroscopic evidence of native liver regeneration $(n=5)$ or significant psychiatric history $(n=2)$.

All patients received $\mathrm{ABO}$ matched left $(n=2)$ or right $(n=5)$ auxiliary grafts. Median duration of surgery was 8.5 (7.3-10 hours), with blood loss of $8 \cdot 31$ (4.6-14.6 litres). Posttransplant, the INR and AST fell progressively in all patients, with median values at day 7 of $1.4(1.0-2 \cdot 4)$ and 108 (78-910 IU/1). Three patients died from sepsis on days 7,10 , and 30. At two weeks post-APOLT, HIDA scans and liver biopsies in the remaining four patients showed partial regeneration of the native lobe in two. At three months, one patient had complete native lobe regeneration, and immunosuppression has since been withdrawn completely.

Although patient selection remains poorly defined, APOLT in ALF is technically feasible and, in some patients, permits native liver regeneration and eventual immunosuppression withdrawal.

\section{$F_{2}$-isoprostanes increase portal pressure}

RICHARD MARLEY, DAVID HARRY, RADHI ANAND, KEVIN MOORE (Academic Department of Medicine, Royal Free Hospital) The $\mathrm{F}_{2}$-isoprostanes (a group of prostaglandin-like compounds, formed by non-enzymatic peroxidation of arachidonic acid), are generated during oxidant stress, and have previously been shown to cause pronounced renal vasoconstriction. Current evidence suggests that these compounds act through a thromboxane-like receptor, as their actions may be blocked by thromboxane receptor antagonists. We have previously shown that these compounds may be generated during hepatic decompensation, and therefore tested the hypothesis that 8-iso- $\mathrm{PGF}_{2 \alpha}$ (a major $\mathrm{F}_{2}$-isoprostane formed in vivo) could increase portal pressure.

The liver from normal or bile duct ligated cirrhotic rats was perfused with KrebsHenseleit buffer in a non-recirulating system at a constant flow rate. 8-iso-PGF infused at $1 \cdot 0,2 \cdot 5$, and $10 \mathrm{~nm} / \mathrm{min}$, and portal pressure monitored continuously.

Infusion of 8-iso-PGF at $_{2 a}$ and 10 $\mathrm{nm} / \mathrm{min}$ into normal liver increased portal pressure from a baseline of $8.2(0.5) \mathrm{mm} \mathrm{Hg}$ (mean (SEM)) to $9.3(0.8)$, and $10.0(0.8)$ $\mathrm{mm} \mathrm{Hg}$ respectively ( $\mathrm{p}<0.05$, Wilcoxon test). At $1 \mathrm{~nm} / \mathrm{min}$, there was no significant change in portal pressure. There was a noticeably increased sensitivity of BDL cirrhotic liver to 8-iso-PGF ${ }_{2 \alpha}$. Portal pressure increased from $11.7(1.0) \mathrm{mm} \mathrm{Hg}$ to $15.9(1.6), 16.4(1.2)$ and $17 \cdot 7(2 \cdot 3) \mathrm{mm} \mathrm{Hg}$ when 8 -iso-PGF $2 \alpha$ was infused at $1 \cdot 0,2 \cdot 5$, and $10 \mathrm{~nm} / \mathrm{min}$ respectively $(p<0.01)$. Preliminary studies have shown that the portal hypertensive effect of 8 iso- $\mathrm{PGF}_{2 \alpha}$ can be blocked by SQ 29,548 , a thromboxane receptor antagonist.

We conclude that 8 -iso- $\mathrm{PGF}_{2 \alpha}$, may cause pronounced changes in the portal pressure in cirrhotic rats. If extrapolated to humans, then episodes of oxidant stress (for example, sepsis or alcoholic binges) may increase the formation of $\mathrm{F}_{2}$-isoprostanes (eg, 8-iso-PGD ${ }_{2 \alpha}$ ), and thus increase portal pressure.

Factors related to early mortality after transjugular intrahepatic portosystemic shunts (TIPS) for uncontrolled variceal haemorrhage

V NIKOLOULOU, D PATCH, P A MCCORMICK, G MATTHEWS, $R$ DICK, A ARMONIS, G WANNAMETHEE, A K BURROUGHS (Hepato-biliary and Liver Transplantation Unit, The Royal Free Hampstead NHS Trust, London NW3) Uncontrolled variceal haemorrhage is the main indication for TIPS. However, mortality is $50 \%$ for this high risk group. We have evaluated clinical and laboratory variables before TIPS to establish predictors of mortality.

Over a three year period 54 patients failed sclerotherapy for acute variceal bleeding and had an emergency TIPS. Failure of sclerotherapy was defined as continued bleeding after two injection sessions $(n=45)$, or bleeding from gastric/ectopic varices $(n=9)$. Some 33 variables were analysed from data available immediately before TIPS

Child/Pugh grade was A:7, B:19, C:28. Four patients continued to bleed after TIPS Twenty four patients died within six weeks. Average follow up was $335 \pm 324$ days. In a multivariate analysis using backward step regression, five factors had independent prognostic value: moderate/severe ascites $(p=0.03)$, requirement for mechanical ventilation $(p=0.003), \log$ WBC $(p=0.0002), \log$ platelet $(p=0.003)$, and $\log$ PTTK $(p=0.004)$.

A prognostic index (PI) score was derived, where presence of moderate/severe ascites, or need for ventilation, scored 1 : $P I=(1 \cdot 34$ (ascites) $)+(1.52$ (ventilation) $)+(2.02 \quad \log$ (WBC) $)-(1.32 \quad \log$ (PLT) $)+(1.95 \quad \log$ (PTTK)). Using this equation, $45 \%(n=10)$ of deaths occurred in the fifth quintile $(P I \geqslant 9 \cdot 3)$, where mortality was $100 \%$.

Patients with uncontrolled variceal haemorrhage have a high mortality despite immediate control of bleeding by TIPS, particularly when associated with markers of advanced liver disease, sepsis, and multi-organ failure. The use of TIPS is probably not justified in this subgroup. The reliability of this prognostic score should be validated prospectively.

Is staging in primary biliary cirrhosis accurate?

M C GARRIDO, S G HUBSCHER (Department of Pathology, University of Birmingham) Our aim was to evaluate sampling variability of liver 
biopsy specimens in patients with primary biliary cirrhosis (PBC).

We examined sections from 50 PBC liver specimens obtained at transplantation. The degree of fibrosis was assessed on a scale of 0-4 using two methods (a) simulated needle biopsy assessing fields approximately the size of a conventional needle biopsy and (b) whole section scanning identifying areas with the least and most severe degrees of fibrosis.

With the method of whole section scanning only 10 cases $(20 \%)$ had a consistent degree of fibrosis in all the sections scanned. By contrast the same stage was assigned in 30 cases $(60 \%)$ examined by the simulated needle biopsy method. Comparison of the results obtained by the two methods showed a discrepancy of one or two stages in 33 cases. This resulted from discovering areas with a lesser degree of fibrosis in whole sections compared with the simulated needle biopsy samples.

There is considerable variation in the degree of fibrosis in livers with PBC even in end-stage specimens obtained at transplantation. Simulated needle specimens give more consistent results. This may be a reflection of the procedure applied when assessing liver needle biopsy specimens where the greatest degree of abnormality is used for determining the stage. Staging of PBC in small needle biopsy specimens is valuable as long as the appearances are interpreted with caution bearing in mind that there is considerable variability in stage.

'True' positive AMA and normal alkaline phosphatase: is this primary biliary cirrhosis (PBC) - 10 years on, the answer is yes

J V METCALF, O F W JAMES, J M PALMER, M F BASSENDINE, D E JONES, H C MITCHISON (Department of Medicine, University of Newcastle upon Tyne NE2 4HH) In 1985 we reported 29 patients with positive AMA and normal LFTs in whom histology was diagnostic/compatible of PBC in 24, normal in two. We now report 10 year follow up. Notes of all 29 were examined, five had died, all other patients were seen again.
Median follow up since first AMA was $14 \cdot 7$ years $(10 \cdot 1-22 \cdot 5)$. Five patients died, none of liver disease, median age 78 (72-83). Twenty four had developed persistent raised alkaline phosphatase, one jaundice (0.9-19 years, median 5.6 after +ve AMA). Twenty two $(75.9 \%)$ developed typical PBC symptoms (pruritus, malaise, persistent upper abdominal pain). Repeat histology was available in 10 (after median 11.4 years). In nine histology was diagnostic/compatible in both biopsy specimens, four had progressed in histological stage. The tenth was normal in both biopsy specimens. Baseline sera were tested against PDC and OGDC (ELISA) in 27. Twenty one were $+\mathrm{ve}, 20$ of these had diagnostic/compatible biopsy specimens. In the six ELISA negative patients only one had a compatible biopsy.

'True' (ELISA +ve) AMA +ve patients with normal LFTs almost certainly have early PBC, confirmed on histology. In this group although disease progression was very slow over $75 \%$ developed typical PBC (symptoms, cholestatic LFTs) within 10 years. 